\title{
Spin-density-functional theory: some open problems and application to inhomogeneous Heisenberg models
}

\author{
K. Capelle and Valter L. Líbero \\ Departamento de Física e Informática \\ Instituto de Física de São Carlos \\ Universidade de São Paulo \\ Caixa Postal 369, 13560-970 São Carlos, SP \\ Brazil
}

September 14, 2018

\begin{abstract}
Spin-density-functional theory (SDFT) is the most widely implemented and applied formulation of density-functional theory. However, it is still finding novel applications, and occasionally encounters unexpected problems. In this paper we first briefly describe a few of the latter, related to issues such as nonuniqueness, noncollinearity, and currents. In the main part we then turn to an example of the former, namely SDFT for the Heisenberg model. It is shown that time-honored concepts of Coulomb DFT, such as the local-density approximation, can be applied to this (and other) model Hamiltonians, too, once the concept of 'density' has been suitably reinterpreted. Local-density-type approximations for the inhomogeneous Heisenberg model are constructed. Numerical applications to finite-size and impurity systems demonstrate that DFT is a computationally efficient and reasonably accurate alternative to conventional methods of statistical mechanics for the Heisenberg model.
\end{abstract}




\section{Introduction}

Density-functional theory (DFT) is widely recognized as a versatile and powerful tool for practical calculations of the electronic structure of matter, on scales ranging from single atoms to extended solids. Its relatively low computational cost makes it attractive as a tool for answering material-specific questions in fields as diverse as quantum chemistry, materials science, and many-body physics [1]. In practice, DFT is most commonly used in its spin-dependent self-consistent-field formulation as Kohn-Sham spin-density-functional theory (SDFT) 2, 3]. Alternative formulations that use other variables in addition to (or instead of) the spin densities, or that proceed without invocation of KohnSham-type equations, are, however, occasionally useful. Some such alternative formulations are described in this contribution. Section 2 gives a brief overview of three recent developments of this type in SDFT, relating to currents, noncollinear spin states and nonuniqueness. In each case a surprising feature of the respective extension of DFT will be highlighted. Section 3 contains a more detailed description of a recent application of SDFT to model Hamiltonians of the Heisenberg type, where results can be obtained without solving Kohn-Sham equations.

\section{Some recent developments in spin-density-func- tional theory}

From a practical point of view, among the most important tasks remaining in the development of SDFT are to obtain ever better approximations for the exchangecorrelation ( $x c$ ) functional, and to learn how to calculate further quantities of physical and chemical interest from the output quantities of SDFT, the spinresolved charge densities. While total energies and related quantities are readily accessible in terms of these densities, many other interesting quantities are not.

\subsection{Electronic currents}

Electronic currents are one example. Such currents are functionals of the charge density, but cannot be calculated as such because this functional is unknown. The current calculated from the Kohn-Sham (KS) orbitals of SDFT is that of the auxiliary noninteracting system, and not guaranteed by the basic theorems of SDFT to be the correct current. The full physical (gauge invariant) current is $\mathbf{j}(\mathbf{r})=\mathbf{j}_{p}(\mathbf{r})+(e / m c) n(\mathbf{r}) \mathbf{A}(\mathbf{r})$, where $\mathbf{A}(\mathbf{r})$ is the vector potential and $\mathbf{j}_{p}(\mathbf{r})$ is the so-called paramagnetic current. The paramagnetic current calculated from the KS orbitals of SDFT is

$$
\mathbf{j}_{p}^{K S}(\mathbf{r})=\frac{\hbar}{2 m i} \sum_{k}\left[\varphi_{k}^{*}(\mathbf{r}) \nabla \varphi_{k}(\mathbf{r})-\varphi_{k}(\mathbf{r}) \nabla \varphi_{k}^{*}(\mathbf{r})\right] \neq \mathbf{j}_{p}[n](\mathbf{r}) .
$$

Current-density functional theory (CDFT) 4, 5] provides a way to calculate the true equilibrium currents by obtaining the orbitals from a modified KS equation 
that features an $x c$ vector potential $\mathbf{A}_{x c}(\mathbf{r})$, chosen such that in terms of these orbitals the above inequality becomes an equality. To achieve this, approximate $x c$ functionals of CDFT must depend on the current density, in addition to the charge and spin densities. This dependence is a complicating factor both in the construction of approximate functionals and in their implementation. In spite of these complications, different ways of constructing CDFT functionals and solving the CDFT KS equations have been explored for atoms, molecules and solids [4, 5, 6, 7, 8, 9, 10, 11. As an alternative to solving the full CDFT equations, usable when current-dependent effects are expected to be small, it has been proposed to cast the CDFT KS equations in the form of SDFT KS equations plus a remainder depending on the $x c$ vector potential $\mathbf{A}_{x c}(\mathbf{r})$, which is then treated perturbatively 12 .

An unexpected feature of CDFT is that, although SDFT and CDFT are independent formulations of DFT for magnetic systems, there is a, somewhat obscure, link between CDFT and SDFT, which may be used to extract information on functionals of one from the other. In Ref. 13 this link is obtained mathematically, but its physical origin is simple to understand: the existence of spin currents of the form $\mathbf{j}_{s}=\nabla \times \mathbf{m}$, where $\mathbf{m}$ is the spin magnetization, implies a connection between the formalisms of SDFT and CDFT, because being a manifestation of the spin degrees of freedom these currents must be describable via SDFT, while as currents they can also be built into the formal framework of CDFT. Some consequences of the resulting connections and consistency relations between SDFT and CDFT are explored in 13. For more details on CDFT we refer to Refs. 4, 15, 6, 7, 8, 9, 10, 11, 12, 13,

\subsection{Noncollinear magnetism}

The standard spin-up spin-down formulation of SDFT is incapable of describing spin configurations that are eigenstates of the square of the total spin operator $\hat{S}^{2}$, but not its $z$-component, $\hat{S}_{z}$. Such noncollinear configurations can arise, e.g., in the presence of spin-orbit coupling, domain walls, external noncollinear fields, or from spontaneous symmetry breaking. Noncollinear ground states are found, e.g., in rare-earth based magnetic systems. In magnetic excitations, noncollinearity is ubiquitious. Consequently, noncollinear formulations both of static [14, 15, 16] and time-dependent [17] DFT have been proposed, but applications are limited to the local-spin-density approximation (LSDA). In trying to describe noncollinear spin states with generalized-gradient approximations (GGAs) or other beyond-LSDA functionals an interesting problem arises.

Due to its origin in the electron liquid, the exchange-correlation energy in the LSDA depends only on the modulus of the spin magnetization $\mathbf{m}(\mathbf{r})$. The corresponding conjugate field, the $x c$ magnetic field

$$
\mathbf{B}_{x c}^{L S D A}(\mathbf{r})=-\frac{\delta E_{x c}^{L S D A}[n,|\mathbf{m}|]}{\delta \mathbf{m}(\mathbf{r})},
$$

is then always parallel to $\mathbf{m}$. Beyond the LSDA, however, the $x c$ magnetic field ceases to be parallel to the spin magnetization. Mathematically this oc- 
curs because the existence of spatial derivatives of any component of $\mathbf{m}$ in the functional leads to additional terms in the derivative that are not parallel to m [18, 19. Intuitively this can be understood because the existence of spatial gradients of the spin density introduces another preferred direction into the problem. In general, the $x c$ magnetic field can thus be decomposed as

$$
\mathbf{B}_{x c}(\mathbf{r})=\mathbf{B}_{x c}^{\|}(\mathbf{r})+\mathbf{B}_{x c}^{\perp}(\mathbf{r}),
$$

where the second contribution is locally perpendicular to the spin magnetization $\mathbf{m}(\mathbf{r})$. This perpendicular $x c$ magnetic field exerts an $x c$ torque $\propto \mathbf{m}(\mathbf{r}) \times \mathbf{B}_{x c}(\mathbf{r})$ on the spin distribution. In the time-dependent case some consequences of this torque were described in 18, but it is present even in the ground state as soon as noncollinearity appears and is treated beyond the LSDA. This subtle effect is little explored in present-day SDFT.

Standard implementations of noncollinear SDFT assume parallelity between $\mathbf{B}_{x c}$ and $\mathbf{m}(\mathbf{r})$, and can thus not be used consistently with GGA-type functionals. What the best way is to treat noncollinearity beyond LSDA is an open question at present. As an alternative to formulations in terms of the spin magnetization, it has been proposed, e.g., to employ the spin-offdiagonal element of the density matrix (the so called 'staggered density' [20]), but this requires the construction of functionals of this new variable, and the solution of more complicated KS equations 21.

\subsection{Nonuniqueness}

At first sight more remote from numerical applications are fundamental issues of SDFT, such as the recently discussed question of nonuniqueness of the SDFT potentials. The question to what extent the effective potentials of SDFT (and other generalized DFTs) are uniquely determined by the densities arises because contrary to what is sometimes claimed in the literature, the standard proofs of the Hohenberg-Kohn theorem (by contradiction 22] and by constrained search 23 ) guarantee only the existence of a one-on-one mapping between densities and wave functions, not densities and potentials. The extension of the mapping to potentials requires, as an additional step, inversion of Schrödinger's equation

to express the potential in terms of the universal operators $\hat{T}$ and $\hat{U}$ and the wave function 23, 24]. For charge-only DFT one has simply

$$
\hat{V}=E_{0}-\frac{(\hat{T}+\hat{U}) \Psi_{0}}{\Psi_{0}},
$$

which shows that the ground-state wave function $\Psi_{0}$ (which is known to be a functional of the density) determines the potential $V$ uniquely up to an additive constant (here the ground-state energy, $E_{0}$ ). Since one always has an additive constant free in the definition of a potential, this shows that the physically relevant part of $\hat{V}$, in particular its variation in space, is fully determined by $\Psi_{0}$ and hence by $n(\mathbf{r})$. Already in the early literature on noncollinear SDFT it was pointed out that this inversion is problematic if one works with 2-component 
spinors and has four potential components $\left(v, B_{x}, B_{y}, B_{z}\right)$ to determine. Von Barth and Hedin gave an explicit example of the resulting nonuniqueness of the SDFT effective potentials, albeit one that only works for a single electron [25].

Recently, examples have been given that show that nonuniqueness is not a pathological feature of single-electron systems, or limited to SDFT, but a general phenomenon, expected to occur whenever one works with more than one density variable [26, 27. In Ref. [27] it was shown how a large class of nonunique potentials is related to the existence of certain types of constants of motion (systematic nonuniqueness) while others arise from special features of the ground state (accidental nonuniqueness). A few simple examples from collinear SDFT were given, illustrating the main features of both types of nonuniqueness. The simplicity of these examples notwithstanding, their existence shows that there cannot be a unique mapping between densities and potentials in generalized DFTs, in addition to the one between densities and wave functions. More complex examples of nonuniqueness were found to be possible in noncollinear situations [26, 28, on lattices [29] or in CDFT 30. The implications of nonuniqueness for practical SDFT calculations are still under study [26, 28, 29, 31, 32. One conclusion that has emerged from this body of work is that the additional mapping between density and potential must be regarded as a special feature of spin unpolarized (charge-only) DFT. Surprisingly, the HK theorem of unpolarized (charge-only) DFT is thus considerably stronger than its counterparts in, e.g., SDFT and CDFT.

\section{Spin density-functional theory for the Heisen- berg model}

Open problems, such as the best way to deal with currents, noncollinearity and nonuniqueness, provide fascinating challenges, but they have never stoped DFT from advancing on the practical side. In a typical application of DFT one deals with the $a b$ initio many-electron Hamiltonian comprising kinetic, potential, and (Coulomb) interaction terms. By means of the Hohenberg-Kohn (HK) theorem and the Kohn-Sham construction this many-body problem is mapped on an auxiliary noninteracting one. The search for good approximations for the exchange-correlation potential appearing in the auxiliary KS Hamiltonian is one of the most active fields of DFT. However, the HK and KS techniques are not restricted to the $a b$ initio Hamiltonian. We can simply regard them as efficient tools for mapping some many-body problem on a simple one-body problem. In many areas of science, most notably perhaps in statistical physics and many-body physics, but occasionally also in quantum chemistry, the fundamental Hamiltonian is replaced by a simpler model Hamiltonian before embarking on any numerical calculation. The list of such simplified Hamiltonians is long, and comprises important models such as those of Ising, Heisenberg and Hubbard. This section is devoted to a description of a recent reformulation and application of SDFT to model Hamiltonians of the Heisenberg type 33, 34. 


\subsection{The Heisenberg model}

Introduced in 1928 by Dirac and Heisenberg [35, 36] and discussed in some detail as a model for ferromagnetic solids in 1932 by van Vleck [37, the Heisenberg model is the paradigmatic model for the magnetism of local magnetic moments $\mathbf{S}_{i}$. In quantum chemistry it has, e.g., been used in the description of the magnetic properties and spin states of conjugated hydrocarbons [38, 39] and a large variety of metallic and organometallic compounds 40, 41, 42, 43.

In its simplest form the Heisenberg model reads

$$
\hat{H}=J \sum_{\langle i j\rangle} \hat{\mathbf{S}}_{i} \cdot \hat{\mathbf{S}}_{j},
$$

where the $\hat{\mathbf{S}}_{i}$ are angular-momentum vector operators satisfying $\hat{\mathbf{S}}^{2}|S m\rangle=$ $\hbar^{2} S(S+1)|S m\rangle$. In accordance with common terminology we refer to the expectation values of $\hat{\mathbf{S}}_{i}$, the magnetic moments $\mathbf{S}_{i}$, as spins, although they may also have an orbital contribution. The sums run over nearest neighbours among the $N$ sites of a lattice of, in principle, arbitrary geometry, and $J$ parametrizes the nearest-neighbor interactions. We see qualitatively that a parallel (ferromagnetic) or antiparallel (antiferromagnetic) alignment of $\mathbf{S}_{i}$ and $\mathbf{S}_{j}$ is favored according to whether $J$ is negative or positive.

In the presence of an external magnetic field $\mathbf{B}_{i}$ the model takes the form

$$
H \hat{(B)}=J \sum_{\langle i j\rangle} \hat{\mathbf{S}}_{i} \cdot \hat{\mathbf{S}}_{j}+\sum_{i} \hat{\mathbf{S}}_{i} \cdot \mathbf{B}_{i},
$$

If $\mathbf{B}_{i}$ and $\mathbf{S}_{i}=\left\langle\hat{\mathbf{S}}_{i}\right\rangle$ are the same everywhere, the model is homogeneous, i.e., all its sites are equivalent. In all other cases it is inhomogeneous. Note that even for $\mathbf{B}_{i}=0$ (no external field) inhomogeneous states are possible. Such solutions may arise because the self-consistent many-body ground state need not preserve all symmetries of the Hamiltonian, or because the Hamiltonian itself comprises inequivalent lattice sites. The latter is the case, e.g., in systems with impurities, or, more generally, with of different types of magnetic atoms.

In spite of its formal simplicity, exact analytical results for the Heisenberg model are known only for homogeneous linear chains with $S=1 / 2$, by means of the Bethe ansatz [44, 45]. For higher spin, higher dimension, or inhomogeneous situations, exact solutions are known only numerically, for small systems (less than $\approx 40$ sites), where the model can be diagonalized exactly by Lanczos techniques. In the presence of inhomogeneities, such as one substitutional spin of value $S^{\prime} \neq S$, no analytical solution is known.

The simplest approximation to the Hamiltonian (5) is the mean-field (Hartreelike) approximation, in which the vector operators $\hat{\mathbf{S}}$ are substituted by classical vector spins $\mathbf{S}$. The mean-field ground-state energy per spin, in units of $J$, is thus easily obtained for a homogeneous infinite system of spins $S$ in $d$ dimensions (with periodic boundary conditions):

$$
e_{0}^{M F}(S, d)=\frac{E_{0}^{M F}(S, d)}{N J}=-d S^{2},
$$


where $d$ is the dimensionality of the linear $(d=1)$, square $(d=2)$ or cubic $(d=3)$ lattice. Other lattice geometries can be dealt with in the same way. An improvement on the mean-field estimate for $e_{0}(S, d)$ is obtained from spin-wave theory [46], according to which

$$
e_{0}^{S W}(S, d)=\frac{E_{0}^{S W}(S, d)}{N J}=-d S^{2}+d^{-1 / 5}\left(\frac{2}{\pi}-1\right) S .
$$

Here we used the scaling hypothesis conjectured in Ref. 33, which accounts for the numerical spin-wave results to three decimal places, to write the results in different dimensionalities in closed form, as a function of $d$. Beyond spin-wave theory, sophisticated numerical many-body techniques, such as the density-matrix renormalization group (DMRG) 47] or Quantum Monte Carlo simulations [48, have been employed to further improve the ground-state energy.

The resulting expressions for $E_{0}$, however, suffer from a major limitation, namely, they are applicable only to homogeneous systems, in which all lattice sites are equivalent. This limitation is restrictive, because in real problems it is very common to find inhomogeneities, induced for instance by external nonuniform magnetic fields, anisotropic crystal-fields, finite-size effects, boundary conditions, local impurities, lattice defects, etc. These rather common situations introduce very significant difficulties in traditional approaches like Bethe ansatz, DMRG, spin-wave theory or even Monte Carlo, by breaking translational symmetry. In order to make progress in dealing with inhomogeneous Heisenberg models we need a many-body technique capable of handling spatial inhomogeneity in large systems. Density-functional theory is our first choice in this regard.

\subsection{Spin-distribution functionals and local-spin approxi- mation}

Density-functional theory [1] 24] offers, with the local-density approximation (LDA), a simple prescription for obtaining ground-state properties of an inhomogeneous system, based on the knowledge of a related homogeneous system. While in ab initio applications of DFT this homogeneous system is the electron liquid, in our context it is the homogeneous Heisenberg model, in which all sites are equivalent.

Ref. 33] shows that the basic ingredients of DFT (in particular the HohenbergKohn theorem [22]) stilll hold for Hamiltonians of the form (6), provided one uses instead of the ground-state density $n(\mathbf{r})$ the ground-state expectation value of the spin vectors, $\mathbf{S}_{i}=\left\langle\Psi\left|\mathbf{S}_{i}\right| \Psi\right\rangle$. The Heisenberg-model Hohenberg-Kohn theorem then states that the ground-state expectation value of any observable $\hat{O}$ is a functional of the distribution $\mathbf{S}_{i}$, i.e., $O\left[\mathbf{S}_{i}\right]=\left\langle\Psi\left[\mathbf{S}_{i}\right]|\hat{O}| \Psi\left[\mathbf{S}_{i}\right]\right\rangle$. In this Heisenberg-model DFT the above mean-field expressions take the place of the Hartree term in Coulomb DFT, and the correlation energy $e_{c}(S)$ is defined as the difference $e_{0}(S)-e_{0}^{M F}(S)$, where $e_{0}(S)$ is the full ground-state energy. From 
the above, the spin-wave approximation for $e_{c}(S)$ is thus

$$
e_{c}^{S W}=e_{0}^{S W}-e_{0}^{M F}=d^{-1 / 5}\left(\frac{2}{\pi}-1\right) S .
$$

The Heisenberg-model Hohenberg-Kohn theorem, proved in 33, guarantees that in inhomogeneous systems the total energy is a functional of the spatial distribution of the classical vectors $\mathbf{S}_{i}$. Hence

$$
E_{0}=E_{0}\left[\mathbf{S}_{i}\right]=E_{0}^{M F}\left[\mathbf{S}_{i}\right]+E_{c}\left[\mathbf{S}_{i}\right] .
$$

Since the mean-field term is already a functional of $\mathbf{S}_{i}$, we just need an approximation for the functional $E_{c}\left[\mathbf{S}_{i}\right]$. This is obtained using a local-spin approximation (LSA), patterned after the conventional LDA of Coulomb DFT. The LSA consists in replacing locally the variable $S$ by $S_{i}$ in $e_{c}(S)$ :

$$
E_{c}\left[\mathbf{S}_{i}\right] \approx E_{c}\left[\mathbf{S}_{i}\right]^{L S A}=\left.\sum_{i}^{N} e_{c}(S)\right|_{S \rightarrow\left|\mathbf{S}_{i}\right|} .
$$

Using the above spin-wave approximation for $e_{c}(S)$ we obtain, e.g.,

$$
E_{c}^{L S A-S W}\left[\mathbf{S}_{i}, d, J\right]=d^{-1 / 5} J\left(\frac{2}{\pi}-1\right) \sum_{i=1}^{N}\left|\mathbf{S}_{i}\right|,
$$

and thus the ground-state energy functional in this approximation is

$$
E_{0}^{L S A-S W}\left[\mathbf{S}_{i}, d, J\right]=J \sum_{\langle i j\rangle} \mathbf{S}_{i} \cdot \mathbf{S}_{j}+d^{-1 / 5} J\left(\frac{2}{\pi}-1\right) \sum_{i}\left|\mathbf{S}_{i}\right|
$$

More sophisticated expressions, based on fully numerical evaluation of $e_{0}(S)$ are also available [33, but in first applications the simple approximation (13) turned out to be already reasonably accurate 34 .

As always in dealing with LDA-type functionals one must distinguish two sources of error. One is the LDA itself, the other the quality of the description of the underlying homogeneous reference system. In Coulomb DFT several alternative parametrizations of the LDA are available [25, 49, which differ considerably in form, and slightly in results. In the present context, too, one can construct various LSA functionals, depending on the level of sophistication employed in solving the uniform Heisenberg model. For example, for an infinite linear chain of spin $S=1 / 2$ we have from the above spin-wave based LSA $e_{0} /(J N)=-0.25-0.181690=-0.431690$, which is $2.6 \%$ from the exact Bethe ansatz value -0.443147 . Interestingly, we find this same margin of deviation also when the LSA is used for inhomogeneous situations (see below). This seems to indicate that the LSA concept as such is quite reliable for Heisenberg models.

From the above, it should have become clear that the formalism of DFT for the Heisenberg model is built in complete parallelity to that of Coulomb DFT 
(or that for the Hubbard model [50]). One interesting difference is that within spin-wave theory the dimension dependence of the functional is approximately known. This is very different from the ab initio case, in which nothing is known about this dependence. In general, however, one may expect that LSA for the Heisenberg model shares the virtues and defects of its close cousin, the LDA for the Coulomb problem: efficient access to large and inhomogeneous systems on one side, offset by moderate accuracy on the other. In the next subsection we demonstrate these aspects by analysing two interesting inhomogeneous models within LSA. (Other applications of the above functionals can be found in Ref. 34.)

\subsection{Applications to inhomogeneous Heisenberg models: boundaries and impurities}

The usual DFT prescription for obtaining ground-state energies is to solve KohnSham equations. These equations, however, were originally [2] introduced to deal with the kinetic-energy term in the Coulomb Hamiltonian. The Heisenberg model does not have such a term, and direct minimization of the energy functional is much simpler than indirect minimization via self-consistency (Kohn-Sham) equations. In practice we just need to minimize the total energy of the system, written as a functional of the spin distribution, with respect to $\mathbf{S}_{i}$. This already represents a considerable improvement over the mean-field approximation with no extra computational cost; minimization of $E_{0}^{L S A-S W}\left[\mathbf{S}_{i}\right]$ is no more complicated than that of $E_{0}^{M F}\left[\mathbf{S}_{i}\right]$.

Lets first consider a finite ring of $N=16$ spins $S=1 / 2$, with periodic boundary conditions. The mean field approximation yields $e_{0}=-0.25$, while the $L S A-S W$ approximation gives $e_{0}=-0.43169$, about $3 \%$ from the exact value -0.44639 obtained by direct diagonalization. Let us now replace any spin of the ring by an impurity spin $S_{I}$. This slight modification is enought to challenge any analytical approach, but since the ring is not too big, it is still possible to numerically diagonalize $\hat{H}$ in order to get the exact ground state. Figure 1 shows how the ground-state energy depends on $S_{I}$, and compares the values obtained by mean-field and LSA-SW calculations with the numerically exact ones. Up to $S_{I}=2.5$ the deviation from the exact values are of order $3 \%$. Above this value the deviation increases, showing that the LSA approximation is not good for large impurity spin (corresponding to a very rapid variation of local magnetic properties). For impurity spins up to $S_{I}=5 / 2$, on the other hand, LSA-SW is seen to provide a substantial improvement on the mean-field approximation. Unlike the exact calculations, LSA-SW can easily be extended to systems with more impurities and more sites. It may thus provide a convenient way of estimating (and improving on) the error of the mean-field approximation in more complicated systems.

Next we consider $4 \times 4$ square lattices with an impurity of spin $S_{I}$ at the corner, at one side, or in the interior of the lattice. Here we use open boundary condition and thus even without impurity the systems are inhomogeneous. Results are collected in Tab. 1. The first column is for $S_{I}=1 / 2$, i.e., for the uniform 
system, and the value listed differs $6.6 \%$ from the exact value $e_{0}=-0.574325$ obtained from numerical diagonalization; we believe the values for other impurity spins are within this accuracy, although no exact values are available, as far as we know. Besides the obvious energetic tendency of higher impurity-spin to prefer the interior of the lattice to its surface, which is already visible in the mean-field results, these data also incorporate less trivial correlation effects: In mean-field theory, for example, a spin $3 / 2$ at the corner of the square is degenerate with a spin 1 in the interior of the lattice $\left(e_{0}^{M F}=-0.4375\right.$ in both cases). This degeneracy is broken by correlation effects. Similarly, mean-field theory predicts degeneracy between a spin $5 / 2$ at one side and a spin 2 in the interior $\left(e_{0}^{M F}=-0.5625\right.$ for both cases). Again this degeneracy is removed by including correlations.

\section{Summary}

Kohn-Sham spin-density functional theory is doubtlessly the most widely used formulation of density-functional theory. However, in spite of its great popularity and usefulness some interesting fundamental questions still remain open. The existence of spin currents, providing a link between SDFT and CDFT; the existence of an exchange-correlation torque appearing in noncollinear spin configurations; and the nonuniqueness of the potentials of SDFT and other generalized DFTs are examples of the kind of surprise the SDFT formalism still holds.

Such open questions notwithstanding, the utility of (S)DFT is extending even into areas remote from $a b$ initio calculations. Recognition that the basic tools and concepts of DFT - such as the Hohenberg-Kohn theorem, and the local-density approximation - are not restricted to the original Coulomb problem allows one to apply these tools and concepts also to many other interesting inhomogeneous model Hamiltonians. In particular, density-functional theory together with results from mean-field and spin-wave theory provide a simple manner to obtain estimates of the ground-state energy for spin distributions described by the inhomogeneous Heisenberg model. This is here illustrated by results for a finite ring and a $4 \times 4$ lattice, both with substitutional impurity spins $S_{I}=1,3 / 2,2$ or $5 / 2$. While ring calculations may find applications in modelling the spin states of hydrocarbons, the square-lattice data illustrate a way in which LSA can be useful in nanomagnetism: To predict the structure of self-assembled magnetic nanostructures it is clearly important to know which lattice sites are degenerate in mean-field theory, and which of these degeneracies are removed by correlations.

This work was supported by FAPESP and CNPq. We thank P. E. G. Assis, and F. C. Alcaraz for useful discussions and collaboration on the Heisenberg model. K.C. thanks L. N. Oliveira, E. K. U. Gross and G. Vignale for useful discussions and collaborations on various aspects of spin-density-functional theory. 


\section{References}

[1] W. Kohn, Rev. Mod. Phys. 71, 1253 (1999).

[2] W. Kohn and L. J. Sham, Phys. Rev. 140, A1133 (1965).

[3] O. Gunnarsson and B. Lundqvist, Phys. Rev. B 13, 4274 (1976).

[4] G. Vignale and M. Rasolt, Phys. Rev. Lett. 59, 2360 (1987).

[5] G. Vignale and M. Rasolt, Phys. Rev. B 37, 10685 (1988).

[6] M. Ferconi and G. Vignale, Phys. Rev. B 50, 14722 (1997).

[7] M. Battocletti, H. Ebert, and E. K. U. Gross, Europhys. Lett. 40, 545 (1997).

[8] A. M. Lee, N. C. Handy, and S. M. Colwell, J. Chem. Phys. 103, 10095 (1996).

[9] K. Capelle, Phys. Rev. B 65, 100515 (2002).

[10] A. Wensauer and U. Rössler, Phys. Rev. B 69, 155302 (2004)

[11] (a) E. Orestes, T. Marcasso and K. Capelle, Phys. Rev. A 68, 022105 (2003). (b) E. Orestes, A. B. F. da Silva and K. Capelle, Int. J. Quantum Chem. 103, 516 (2005).

[12] K. Capelle, Phys. Rev. A 60, 733 (1999).

[13] K. Capelle and E. K. U. Gross, Phys. Rev. Lett. 78, 1872 (1997).

[14] L. Nordström and D. Singh, Phys. Rev. Lett. 76, 4420 (1996).

[15] J. Sticht, K.-H. Höck, and J. Kübler, J. Phys. Condens. Matter 1, 8155 (1989).

[16] L. M. Sandratskii, Adv. Phys. 47, 91 (1998).

[17] F. Wang and T. Ziegler, J. Chem. Phys. 122, 074109 (2005).

[18] K. Capelle, G. Vignale, and B. L. Gyorffy, Phys. Rev. Lett. 87, 206403 (2001).

[19] K. Capelle and B. L. Gyorffy, Europhys. Lett. 61, 354 (2003)

[20] K. Capelle and L. N. Oliveira, Europhys. Lett. 49, 376 (2000).

[21] K. Capelle and L. N. Oliveira, Phys. Rev. B 61, 15228 (2000).

[22] P. Hohenberg and W. Kohn, Phys. Rev. 136, B864 (1964).

[23] M. Levy, Adv. Quant. Chem. 21, 69 (1990). 
[24] R. M. Dreizler and E. K. U. Gross, Density Functional Theory (Springer, Berlin, 1990).

[25] U. von Barth and L. Hedin, J. Phys. C 5, 1629 (1972).

[26] H. Eschrig and W. E. Pickett, Solid State Commun. 118, 123 (2001).

[27] K. Capelle and G. Vignale, Phys. Rev. Lett. 86, 5546 (2001).

[28] N. Gidopolous in The fundamentals of density matrix and density functional theory in atoms, molecules, and solids, N. Gidopoulos and S. Wilson eds. (Kluwer Series 'Progress in Theoretical Chemistry and Physics', 2003).

[29] C. A. Ullrich, to be published (2005).

[30] K. Capelle and G. Vignale, Phys. Rev. B 65, 113106 (2002).

[31] N. Argaman and G. Makov, Phys. Rev. B 66, 052413 (2002).

[32] W. Kohn, A. Savin and C. A. Ullrich, Int. J. Quantum Chem. 100, 20 (2004).

[33] Valter L. Líbero and K. Capelle, Phys. Rev. B 68, 024423 (2003).

[34] P. E. G. Assis, Valter L. Líbero and K. Capelle, Phys. Rev. B. 71, 052402 (2005).

[35] P. A. M. Dirac, Proc. Roy. Soc. 112A, 661 (1926), Proc. Roy. Soc. 123A, 714 (1929).

[36] W. Heisenberg, Z. Phys. 38, 441 (1926), Z. Phys. 49, 619 (1928).

[37] J. H. Van Vleck, The Theory of Electric and Magnetic Susceptibilities, Claredon Press, Oxford.

[38] N. Guihery, N. Ben Amor, D. Maynau and J. P. Malrieu, J. Chem. Phys. 104, 3701 (1996).

[39] M. Said, D. Maynau, J. P. Malrieu and M. A. Garcia Bach, J. Am. Chem. Soc. 106, 571 (1984).

[40] A. L. Tchougreeff, J. Chem. Phys. 96, 6026 (1992).

[41] C. Kollmar and O. Kahn, J. Chem. Phys. 98, 453 (1993).

[42] M. Matusiewicz, M. Czerwinski, J. Kasperczyk and I. V. Kityk, J. Chem. Phys. 111, 6446 (1999).

[43] J. H. Luscombe, M. Luban and F. Borsa, J. Chem. Phys. 108, 7266 (1998).

[44] H. Bethe, Z. Phys. 71, 205 (1931).

[45] L. Hulthen, Arkiv Mat. Astron. Fysik 26A, No.11, 1 (1938). 
[46] P. W. Anderson, Phys. Rev. 86, 694 (1952).

[47] J. Lou, S. Qin, T.-K. Ng and Z. Su, Phys. Rev. B 65, 104401 (2002).

[48] K. H. Hoglund and A. W. Sandvik, Phys. Rev. Lett. 91, 077204 (2003).

[49] O. Gunnarsson and B. Lundqvist, Phys. Rev. B 13, 4274 (1976). S. H. Vosko, L. Wilk, and M. Nusair, Can. J. Phys. 58, 1200 (1980). J. P. Perdew and A. Zunger, Phys. Rev. B 23, 5048 (1981). J. P. Perdew and Y. Wang, Phys. Rev. B 45, 13244 (1993).

[50] N. A. Lima, M. F. Silva, L. N. Oliveira and K. Capelle, Phys. Rev. Lett. 90, 146402 (2003). 
Table 1: LSA-SW ground-state energy $-E_{0} / N J$ of a $4 \times 4$ square lattice of $S=1 / 2$ sites with a substitutional impurity of spin $S_{I}$ at the corner (four equivalent positions), on a side (eight equivalent positions) or in the interior of the lattice (four equivalent positions).

\begin{tabular}{r|c|c|c|c|c} 
& $1 / 2$ & 1 & $3 / 2$ & 2 & $5 / 2$ \\
\hline corner & 0.612 & 0.653 & 0.694 & 0.735 & 0.776 \\
side & 0.612 & 0.669 & 0.726 & 0.782 & 0.839 \\
interior & 0.612 & 0.684 & 0.757 & 0.829 & 0.902
\end{tabular}




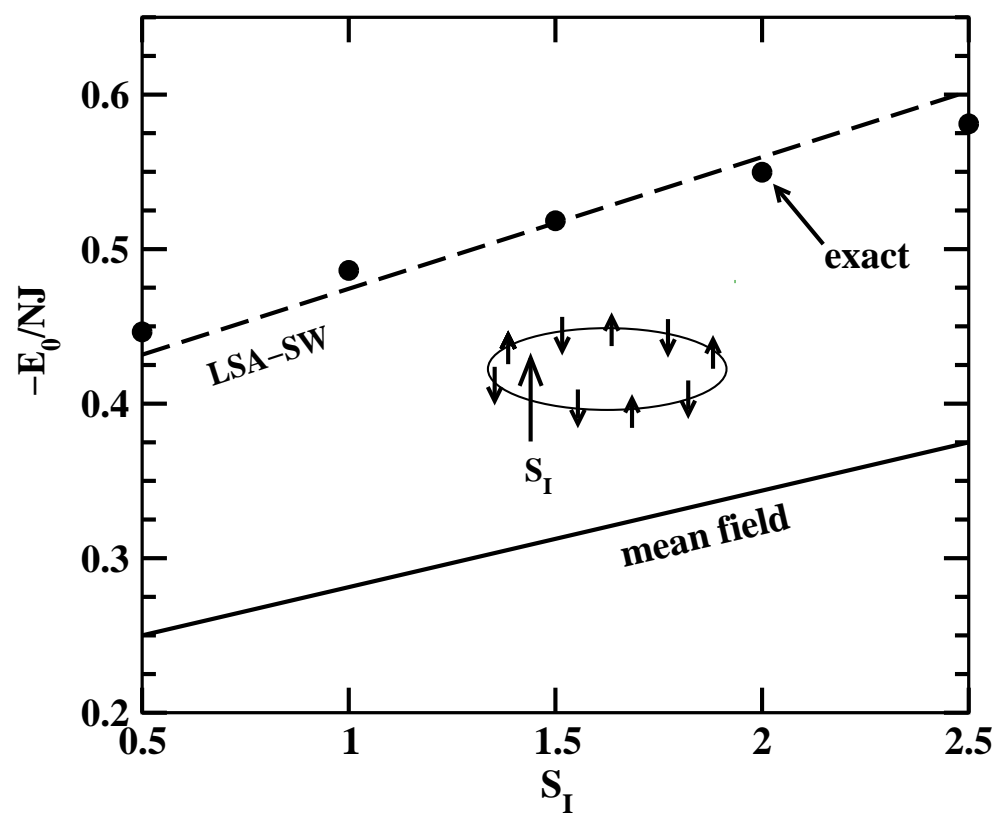

15

Figure 1: Per-site ground-state energy of a ring of $N=16$ spin $S=1 / 2$ sites as a function of the impurity spin $S_{I}$. The LSA results, based on spin-wave theory, (dashed line) deviate from the exact ones (circles) by about $3 \%$. The mean field approximation (continuous line) deviates by up to $50 \%$. The inset illustrates the geometry under study, for the case of $N=10$. 